\title{
Plasmon-enhanced erbium luminescence
}

\author{
H. Mertens ${ }^{\text {a) }}$ and A. Polman \\ Center for Nanophotonics, FOM Institute for Atomic and Molecular Physics, Kruislaan 407, \\ 1098 SJ Amsterdam, The Netherlands
}

(Received 25 August 2006; accepted 6 October 2006; published online 21 November 2006)

\begin{abstract}
It is demonstrated that the photoluminescence intensity of optically active erbium ions positioned in close proximity of anisotropic Ag nanoparticles is significantly enhanced if the nanoparticles support plasmon modes that are resonant with the erbium emission. In addition, the photoluminescence intensity enhancement is found to be polarized corresponding to polarization of these plasmon modes. Both observations demonstrate that the photoluminescence enhancement is due to coupling of the $\mathrm{Er}^{3+}{ }^{4} I_{13 / 2}-{ }^{4} I_{15 / 2}$ transition dipoles with plasmon modes in the $\mathrm{Ag}$ nanoparticles. As this coupling mechanism is known to affect the emission rate, metal nanoparticles provide an opportunity to reduce the effect of temperature or concentration quench processes that are known to occur in a wide range of erbium-doped materials. (C) 2006 American Institute of Physics. [DOI: 10.1063/1.2392827]
\end{abstract}

Erbium-doped materials are of great interest in optoelectronics due to the $\mathrm{Er}^{3+}$ intra- $4 f$ emission at $1.54 \mu \mathrm{m}$, a standard telecommunications wavelength. Dielectrics doped with erbium can be used to fabricate planar optical amplifiers ${ }^{1}$ and lasers, ${ }^{2}$ as well as light-emitting devices. ${ }^{3}$ Erbium doping of silicon enables the realization of optoelectronic devices based on the most widely used semiconductor in microelectronics.

For all Er-doped materials, however, a practical limitation is that due to the small oscillator strength of the $\mathrm{Er}^{3+}$ intra- $4 f$ transition at $1.54 \mu \mathrm{m}\left({ }^{4} I_{13 / 2}-{ }^{4} I_{15 / 2}\right)$, loss processes can easily dominate the deexcitation of the ${ }^{4} I_{13 / 2}$ level. For instance, in Er-doped dielectrics, the Er concentration needs to be kept low to avoid concentration quenching and cooperative upconversion. ${ }^{4}$ In Er-doped $\mathrm{Si}$, temperature quenching is the main constraint for device applications. ${ }^{5}$ It is caused by phonon-assisted energy backtransfer and Auger interactions with free carriers. ${ }^{6}$ Engineering of the Er sites, for example, by oxygen codoping, ${ }^{5}$ as well as device engineering ${ }^{7}$ have not reduced the significance of these nonradiative processes sufficiently in order to turn Er-doped Si into a practical optoelectronic material.

An alternative approach to diminish the effect of quenching, which is different from limiting nonradiative processes, consists of enhancing the spontaneous emission rate of the $\mathrm{Er}^{3+}{ }^{4} I_{13 / 2} \rightarrow{ }^{4} I_{15 / 2}$ transition by modifying the dielectric environment. ${ }^{8}$ Very strong effects of the environment on the emission rate are predicted for emitters that are positioned in close proximity of metal nanoparticles. ${ }^{9}$ If a plasmon mode, i.e., a collective conduction electron oscillation, ${ }^{10}$ in a metal nanoparticle is resonant with an optical transition, the electromagnetic coupling between transition dipole and plasmon mode can result in an enhancement of the radiative decay rate by several orders of magnitude for emitternanoparticle separation distances less than $\sim 20 \mathrm{~nm}$.

Most of the recent work on interactions between emitters and metal nanoparticles has been done with dyes or quantum dots that emit at wavelengths between 500 and $900 \mathrm{~nm}$ (see,

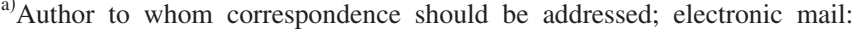
mertens@amolf.nl
}

e.g., Refs. 11-13), a spectral range for which it is relatively easy to obtain resonant plasmon modes. However, enhancing the $\mathrm{Er}^{3+}$ radiative decay rate at $1.54 \mu \mathrm{m}$ by employing resonant plasmon modes could be very attractive as well, especially in view of the opportunity to reduce the significance of various quench processes, including temperature quenching of $\mathrm{Er}$ in $\mathrm{Si}$.

In this letter, we report on the coupling of optically active $\mathrm{Er}^{3+}$ ions with plasmon modes in $\mathrm{Ag}$ nanoparticles. Firstly, it is shown that by engineering Ag nanoparticles with a strong geometrical anisotropy, plasmon modes with a resonance wavelength of $1.5 \mu \mathrm{m}$ can be obtained. Secondly, it is shown that the photoluminescence (PL) intensity of $\mathrm{Er}^{3+}$ ions positioned close to these nanoparticles is significantly enhanced. The dependences of the PL enhancement on both nanoparticle geometry and polarization demonstrate that the PL enhancement is due to coupling of the $\mathrm{Er}^{3+}{ }^{4} I_{13 / 2}-{ }^{4} I_{15 / 2}$ transition dipoles with plasmon modes in the $\mathrm{Ag}$ nanoparticles.

The sample preparation was done in the following way. First, a 1-mm-thick $\mathrm{SiO}_{2}$ substrate was covered with a 45-nm-thick sacrificial Al layer. Subsequently, the sample was implanted with $1.2 \times 10^{16} \mathrm{Er}$ ions $/ \mathrm{cm}^{2}$ at $150 \mathrm{keV}$, after which the Al layer was etched off in a 1:10 solution of $\mathrm{KOH}$ in water. As a result, the $\mathrm{SiO}_{2}$ substrate was doped with $9 \times 10^{15} \mathrm{Er}$ ions $/ \mathrm{cm}^{2}$ in the top $50 \mathrm{~nm}$, with a maximum concentration of 4 at. $\%$ at a depth of $10 \mathrm{~nm}$. In order to obtain a model system in which the Er luminescence is quenched considerably, the sample was not annealed. The PL lifetime at $1.54 \mu \mathrm{m}$ was less than $0.5 \mathrm{~ms}$, which indicates that the internal quantum efficiency for emission is smaller than $\sim 3 \%$, since the radiative lifetime of $\mathrm{Er}^{3+}$ in $\mathrm{SiO}_{2}$ is $18 \mathrm{~ms}^{14}$

On top of the Er-doped $\mathrm{SiO}_{2}$ substrate, $\mathrm{Ag}$ nanoparticles were fabricated using electron-beam lithography, Ag evaporation, and lift-off. Silver was chosen because this metal exhibits the lowest Ohmic damping at visible and infrared frequencies, and thus gives rise to the highest electromagnetic field enhancements. ${ }^{10}$ The resulting structure is schematically depicted in Fig. 1(a), and scanning electron micrograph images of the arrays are displayed in Fig. 1(b). From top left to bottom right, the Ag nanoparticle length $l$ is increased from 
(a)

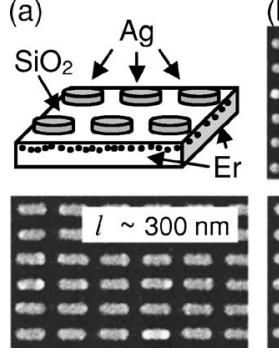

(b)

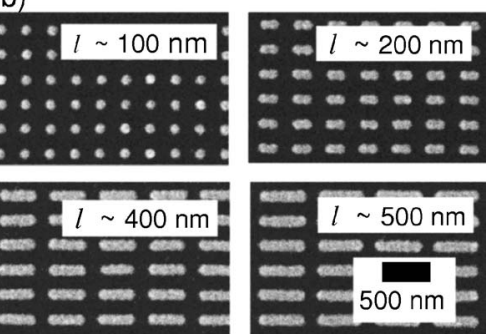

FIG. 1. (a) Schematic representation of the sample consisting of a $\mathrm{SiO}_{2}$ substrate, which is doped with Er ions in the upper $50 \mathrm{~nm}$, with an array of Ag nanoparticles on top. (b) Scanning electron micrographs of five arrays of $\mathrm{Ag}$ nanoparticles. The nanoparticle length $l$ is increased from $\sim 100$ to $\sim 500 \mathrm{~nm}$, while the width is kept constant at $\sim 100 \mathrm{~nm}$. The height of the nanoparticles is $20 \mathrm{~nm}$ for all arrays.

$\sim 100$ to $\sim 600 \mathrm{~nm}$, while the nanoparticle width is kept nearly constant at $\sim 100 \mathrm{~nm}$. The height of all $\mathrm{Ag}$ nanoparticles is $20 \mathrm{~nm}$, so that the smallest nanoparticles can be considered as circular disks, whereas all the other nanoparticles are elongated disks.

The degree of geometrical anisotropy strongly influences the resonance frequencies of the nanoparticle plasmon modes, as can be seen in Fig. 2, which displays the transmission spectra of the arrays. These transmission spectra were measured at normal incidence with unpolarized light by illumination through a $60 \times$ microscope objective $(\mathrm{NA}=0.8)$ and collection through a $20 \times$ microscopy objective $(\mathrm{NA}=0.4)$. The transmission through each $\mathrm{Ag}$ nanoparticle array was normalized by the transmission through an unpatterned reference area.

As can be seen in Fig. 2, the transmission spectrum of the circular disks $(l \sim 100 \mathrm{~nm})$ exhibits a broad dip centered at $2.1 \mathrm{eV}$, which can be attributed to absorption and scattering due to excitation of dipole plasmon modes in the $\mathrm{Ag}$ nanoparticles. ${ }^{10}$ The spectral position of this dip depends on nanoparticle size and shape, the refractive index of the embedding media, and on coupling between the nanoparticles. ${ }^{10}$ Note that in the Er emission band around $0.81 \mathrm{eV}$, which is indicated by the vertical gray band, the transmission for this array is nearly unity.

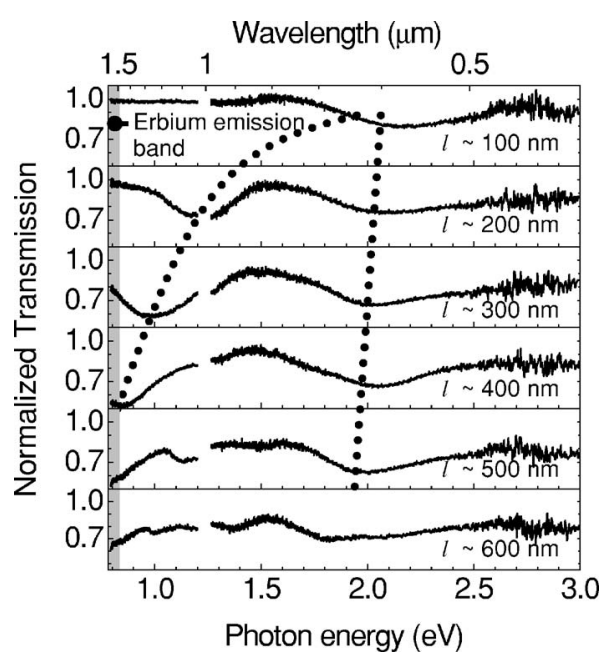

FIG. 2. Transmission spectra of six Ag nanoparticle arrays, with the nanoparticle length $l$ increasing from $\sim 100$ to $\sim 600 \mathrm{~nm}$. The dotted lines indicate the evolution of the transmission dips as function of the nanoparticle length. length.
Downloaded 18 Dec 2006 to 192.87.154.124. Redistribution subject to AlP license or copyright, see http://apl.aip.org/apl/copyright.jsp

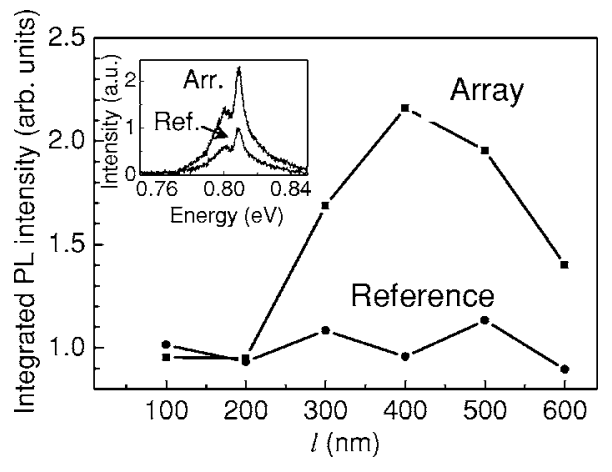

FIG. 3. Integrated PL intensity at $1.54 \mu \mathrm{m}$ as function of nanoparticle length $l$ for six Ag nanoparticle arrays. Data measured both on the array and on the unpatterned reference area are shown. The inset shows two typical Er emission spectra. Pump wavelength: $532 \mathrm{~nm}$.

For the smallest elongated nanoparticles $(l \sim 200 \mathrm{~nm})$, the transmission spectrum is characterized by two dips. This is due to the fact that the degeneracy for the two polarizations is lifted. The low-energy dip is associated with the longitudinal plasmon mode (parallel to the long axis), whereas the high-energy dip is associated with the transverse plasmon mode (perpendicular to the long axis). ${ }^{10}$ The low-energy dip can be shifted towards the erbium emission band by increasing the anisotropy, as can be seen for $l \sim 300 \mathrm{~nm}$ and $l$ $\sim 400 \mathrm{~nm}$, and as is indicated by the left dotted line in Fig. 2. By further elongation, the low-energy dip shifts out of the detector range. Additional features appearing between 1.0 and $1.5 \mathrm{eV}$ are attributed to the excitation of higher-order plasmon modes.

In order to study the influence of the metal nanoparticles on the Er luminescence, PL spectra were acquired under excitation from a frequency-doubled neodymium-doped yttrium aluminum garnet laser operating at $\lambda_{\mathrm{exc}}=532 \mathrm{~nm}$ focused through $60 \times$ microscopy objective $(\mathrm{NA}=0.8)$ from above the sample [see Fig. 1(a)] to a 10- $\mu$ m-diameter spot. The PL intensity was collected from the bottom with $20 \times$ microscopy objective $(\mathrm{NA}=0.4)$ and recorded using a charge-coupled device detector in conjunction with a $30 \mathrm{~cm}$ focal length grating spectrograph. A dichroic filter was used to eliminate incident laser light from the detector. Reference PL spectra were obtained from areas close to the arrays where the Er implanted area was not covered with Ag nanoparticles for identical pump powers (see Ref. 13 for more information on the measurement configuration).

Figure 3 shows the integrated Er PL intensity obtained at $1.54 \mu \mathrm{m}$ for six arrays with increasing nanoparticle length $l$ ( $l$ from 100 to $600 \mathrm{~nm}$ ). In addition, the integrated PL intensity measured on nearby areas without nanoparticles is displayed as well. Two typical PL spectra for array and reference are shown in the inset. As can be seen in Fig. 3, the integrated PL intensities for array and reference are nearly identical for both $l \sim 100 \mathrm{~nm}$ and $l \sim 200 \mathrm{~nm}$. Note that these data points correspond to the arrays for which the transmission in the Er emission band is practically unity (see Fig. 2). For longer nanoparticles, for which the low-energy transmission dip exhibits spectral overlap with the erbium emission band, the PL intensity on the array is enhanced compared to the reference area. The largest enhancement is observed for the nanoparticle length $(l \sim 400 \mathrm{~nm})$ for which the lowenergy transmission dip has the best overlap with the Er 


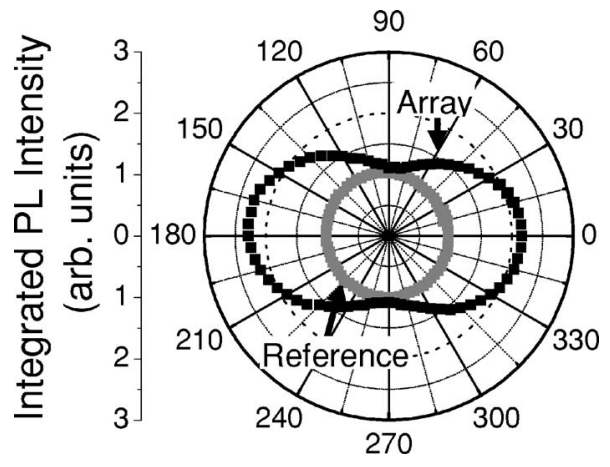

FIG. 4. Polar plot of the PL intensity for array $(l \sim 400 \mathrm{~nm})$ and reference, measured as function of the polarization angle $\theta$ of the emitted light relative to the long axis of the Ag nanoparticles. Pump wavelength: $532 \mathrm{~nm}$.

the longitudinal plasmon mode, it can be concluded that the observed PL enhancement is due to the coupling of the $\mathrm{Er}^{3+}$ ${ }^{4} I_{13 / 2}-{ }^{4} I_{15 / 2}$ transition dipoles with longitudinal plasmon modes in the nanoparticles. The close correspondence between array and reference measurements for both $l \sim 100 \mathrm{~nm}$ and $l \sim 200 \mathrm{~nm}$, for which no plasmon mode is resonant with the emission, indicates that in the present configuration the measured PL intensity is not strongly affected by the potential difference in pump conditions between array and reference measurements.

A second demonstration that the observed PL enhancement is due to the coupling of $\mathrm{Er}^{3+}$ ions with plasmon modes is given by polarization-dependent measurements of the PL intensity. These measurements were performed in the same configuration as was used to obtain the data shown in Fig. 3, except that the emitted light was passed through a polarization analyzer before illuminating the detector.

Figure 4(a) shows the PL intensity as function of the polarization angle $\theta$ of the emitted light relative to the long axis of the nanoparticles (in the plane perpendicular to the surface normal) for $l \sim 400 \mathrm{~nm}$. The PL intensity on the unpatterned reference area is polarized isotropically (the circular pattern in the center of the plot), which implies that the PL intensity does not depend on the polarization of the pump light. The PL intensity on the array is clearly enhanced compared to the reference. Moreover, the enhancement is polarized in the direction of the long axis, which corresponds to the polarization direction of the nanoparticle plasmon mode that is resonant at $1.5 \mu \mathrm{m}$. This correspondence demonstrates that the observed PL enhancement is due to coupling of the $\mathrm{Er}^{3+}{ }^{4} I_{13 / 2}-{ }^{4} I_{15 / 2}$ transition dipoles with plasmon modes in the metal nanoparticles.

It can be noted that the profiles in Fig. 4 are not completely symmetric for rotation over $180^{\circ}$. The asymmetry is attributed to a small misalignment of the polarization ana- lyzer. By normalizing the angular profile of the array by the reference data, perfectly symmetric curves are observed (not shown).

The twofold PL enhancement observed is an ensemble average. Much larger local enhancement values are expected close to the sharp corners of the metal nanoparticles. ${ }^{9}$ Utilization of dense arrays of smaller nanoparticles is therefore expected to result in much larger ensemble-averaged enhancements.

In conclusion, we have observed an enhancement of the photoluminescence intensity at $1.54 \mu \mathrm{m}$ of optically active $\mathrm{Er}^{3+}$ ions in $\mathrm{SiO}_{2}$ by coupling to engineered $\mathrm{Ag}$ nanoparticles. The PL enhancement is maximal if the Er emission is resonant with nanoparticle plasmon modes. Moreover, the PL enhancement is polarized corresponding to the polarization of the plasmon modes. Both these observations demonstrate that the $\mathrm{Er}^{3+}{ }^{4} I_{13 / 2}-{ }^{4} I_{15 / 2}$ transition dipoles are coupled with nanoparticle plasmon modes. As this coupling mechanism is known to affect the emission rate, metal nanoparticles may reduce the significance of quench processes of $\mathrm{Er}$ embedded in various host materials, such as concentration quenching and upconversion in Er-doped dielectrics and temperature quenching of $\mathrm{Er}$ in $\mathrm{Si}$.

This work is part of the research program of FOM, which is financially supported by NWO. This work was also partially supported by NANONED.

${ }^{1}$ G. N. van den Hoven, E. Snoeks, A. Polman, C. van Dam, J. W. M. van Uffelen, and M. K. Smit, J. Appl. Phys. 79, 1258 (1996).

${ }^{2}$ A. Polman, B. Min, J. Kalkman, T. J. Kippenberg, and K. J. Vahala, Appl. Phys. Lett. 84, 1037 (2004).

${ }^{3}$ M. E. Castagna, S. Coffa, M. Monaco, A. Muscara, L. Caristia, S. Lorenti, and A. Messina, Mater. Sci. Eng., B 105, 83 (2003).

${ }^{4}$ E. Snoeks, P. G. Kik, and A. Polman, Opt. Mater. (Amsterdam, Neth.) 5, 159 (1996).

${ }^{5}$ S. Coffa, G. Franzó, F. Priolo, A. Polman, and R. Serna, Phys. Rev. B 49, 16313 (1994).

${ }^{6}$ F. Priolo, G. Franzó, S. Coffa, and A. Carnera, Phys. Rev. B 57, 4443 (1998).

${ }^{7}$ S. Coffa, G. Franzó, and F. Priolo, Appl. Phys. Lett. 69, 2077 (1996).

${ }^{8}$ E. Snoeks, A. Lagendijk, and A. Polman, Phys. Rev. Lett. 74, 2459 (1995).

${ }^{9}$ J. Gersten and A. Nitzan, J. Chem. Phys. 75, 1139 (1981).

${ }^{10} \mathrm{U}$. Kreibig and M. Vollmer, Optical Properties of Metal Clusters (Springer, Berlin, 1995), Chap. 2.

${ }^{11}$ S. Kühn, U. Håkanson, L. Rogobete, and V. Sandoghdar, Phys. Rev. Lett. 97, 017402 (2006).

${ }^{12}$ P. Anger, P. Bharadwaj, and L. Novotny, Phys. Rev. Lett. 96, 113002 (2006).

${ }^{13}$ J. J. S. Biteen, N. S. Lewis, H. A. Atwater, H. Mertens, and A. Polman, Appl. Phys. Lett. 88, 131109 (2006).

${ }^{14}$ M. J. A. de Dood, L. H. Slooff, A. Polman, A. Moroz, and A. van Blaaderen, Phys. Rev. A 64, 33807 (2001). 